\title{
Preliminary View of a Smart Technique for Materials Testing in the Laboratory using FBG Sensor
}

\author{
Isah W. Balarabe ${ }^{1,}{ }^{*}$, Mohamad Hisham ${ }^{1}$, Ahmad R. Niraku ${ }^{1}$, and Indra S.H. Harahap ${ }^{1}$ \\ ${ }^{1}$ Universiti Teknologi PETRONAS, Civil and Environmental Engineering Department, 32610 Bandar \\ Seri Iskandar Perak, Malaysia
}

\begin{abstract}
Simple experiments for calibrating Fibre Bragg Grating (FBG) in order to measure strain and temperature have been successfully demonstrated in this study. This will allow convenient use of FBG sensor in the laboratory for measuring quantities. Linear curve fittings were employed for both the strain and temperature, and $R^{2}$ values are determined respectively. The result showed a perfect correlation between FBG, strain and temperature. The approach can be employed with ease, simplicity in an inexpensive manner, in order harness the advantages offered by FBG sensors such as accuracy, precision, speed and high resolution.
\end{abstract}

\section{Introduction}

Fibre optic sensing technologies have gain prospects for use in many disciplines due to its versatility [1]-[4]. Two types of this technology are the most commonly adopted; distributed fibre optics (DFO) and Fibre Brag Grating (FBG) sensing techniques. Recently, a conjuncture helical configuration developed by [5] from a single distributed optical fibre for laboratory characterization of materials have shown good correlation when compared with LVDT apart from the advantage of being immune to effect of lightning and electrical short circuiting which the existing devices are vulnerable to Fibre Bragg Grating (FBG) sensing technique is now being used in the laboratory for measuring quantities such as strain, temperature, acceleration and pressure[6]-[9]. The advantages offered by the sensor; extreme sensitivity, light in weight immune to electromagnetic interference (EMI), resistance to hash environment, multiplexing and multifunctionality have rendered the sensor useful in civil and many other engineering disciplines.

The study intends to elucidate a simple method of calibrating FBG sensor so that the sensor can be adopted to measure strain and temperature. FBG sensor delineates measurements of physical quantities in terms of wavelength, which must be converted by a suitable means to a quantity it is intended to measure as it can be used to measure several quantities.

\footnotetext{
* Corresponding author: balarabe 16005697@utp.edu.my
} 


\subsection{Working principle of FBG sensor}

Recently, Fibre optics sensing technique have shown potentials in laboratory experimentations [6], [10], [11]. An FBG is a distributed Bragg reflector imprinted in a small section of an optical fibre which can reflect a specific wavelength of light and transmitting all the other wavelengths. FBG sensors are formed by engraving an unseen permanent periodic refractive index changes in fibre core. FBG can be uniform, chirped, tilted or superstructure. FBG is capable of transmitting light over a long distance with very little loss of precision [9]. Schematic diagram of FBG sensors working principle is shown in Fig. 1. There are two important components of FBG, the core and the cladding. Gratings are written on the core (inner component) which serves as guide to the light along the optical fibre. The grating is normally written on silica optical fibre of very small in diameter about the size of human hair. Cladding surrounded the core to ensure total internal reflection within the core, and that very small light is lost as the refraction index of the cladding is lower than that of the core. External factors such as heat and pressure will cause a shift in the wavelength of the reflected light; this variation can then be translated in to physical engineering units such as amplitude, strain, and temperature with the help of interrogator. The common FBG data acquisition system can detect a shift in the Bragg wavelength of as small as 1pico meter (10$\left.{ }^{12} \mathrm{~m}\right)$.

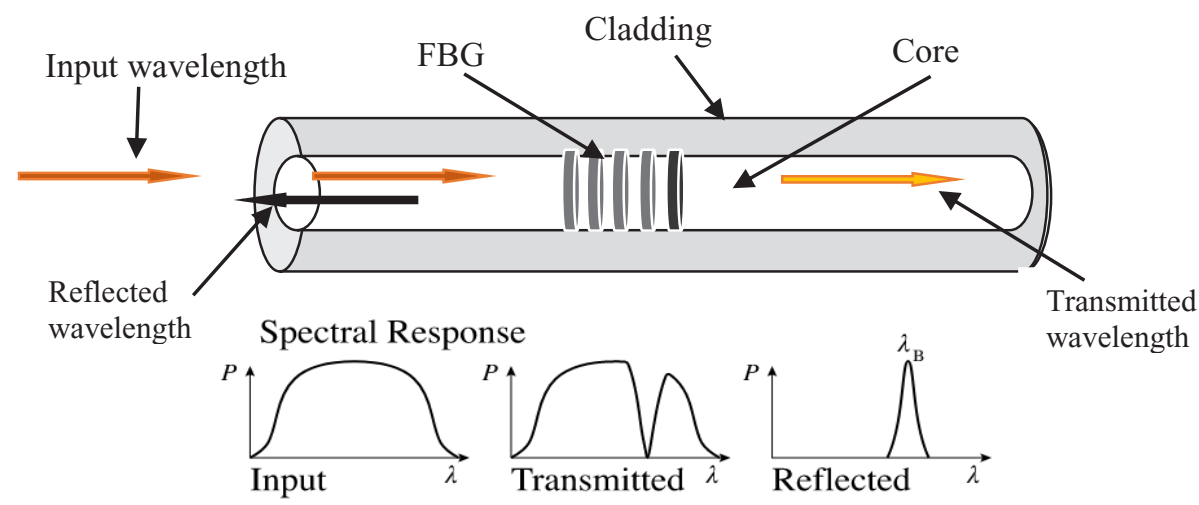

Fig. 1. Schematic view of working principle of FBG sensor.

From Fig. 1, the reflected light's wavelength can be obtained using equation 1.

$$
\lambda_{B}=2 n_{e f f} \Lambda
$$

Where $n_{\text {eff }}$ is the refraction index of the core of the fibre and $\Lambda$ is the grating period of index modulation. FBG is noted to be a thermocouple material and can be affected by temperature. Therefore, during measurement readings and when there is changes in the temperature, a separate temperature FBG sensor (isolated from the influence of measured strain) must be attached to compensate the temperature. Once the temperature is known, the strain can be calculated and the shift in the wavelength $\left(\Delta \lambda_{B}\right)$ during experiment can be determined using equation 2 below [12].

$$
\frac{\Delta \lambda_{B}}{\lambda_{B}}=\left(1-p_{e}\right) \Delta \varepsilon+(\alpha+\xi) \Delta T
$$


where;

$p_{e}=$ effective photo-elastic constant of the fiber core material

$\Delta \varepsilon=$ change in the strain

$\xi$ and $\alpha=$ temperature coefficients

$\Delta T=$ change in temperature.

Generally, when fiber is doped with germanium at room temperature, the value of $\alpha$ and $\xi \cong 0.5 \times 10^{-6} /{ }^{0} \mathrm{C}$ and $7.0 \times 10^{-6} /{ }^{0} \mathrm{C}$. When temperature is constant, equation $2 \mathrm{can}$ be written as:

$$
\frac{\Delta \lambda_{B}}{\lambda_{B}}=k \varepsilon
$$

Where $k$ is a constant which can be determined in the laboratory.

\section{Methodology}

This section covers the detail discussion of the experiments conducted for the temperature and strain calibration using FBG in the laboratory. These experiments were conducted with the aim of establishing control of FBG sensor for the purpose of specimen deformation measurement.

\subsection{FBG sensor temperature calibration}

In this study, temperature calibration was conducted using a heating system consisting a glass box fitted with heating coil and temperature control. The glass box is filled with water, FGB sensor with wavelength of $1569 \mathrm{~nm}$ is immersed in water. The rise in temperature is monitored at an interval of $5{ }^{\circ} \mathrm{C}$ up to $85^{\circ} \mathrm{C}$ and the corresponding FGB readings were taken at the frequency of $1 \mathrm{kHz}$ with the aid of micron optics interrogator. On reaching $85{ }^{\circ} \mathrm{C}$, the decrease in temperature is also monitored at similar interval to obtain a complete cycle of temperature calibration. The selected maximum temperature of $85{ }^{\circ} \mathrm{C}$ is sufficient because the entire experiments would be conducted at room temperature. The setup of the apparatus is illustrated in Fig.2.

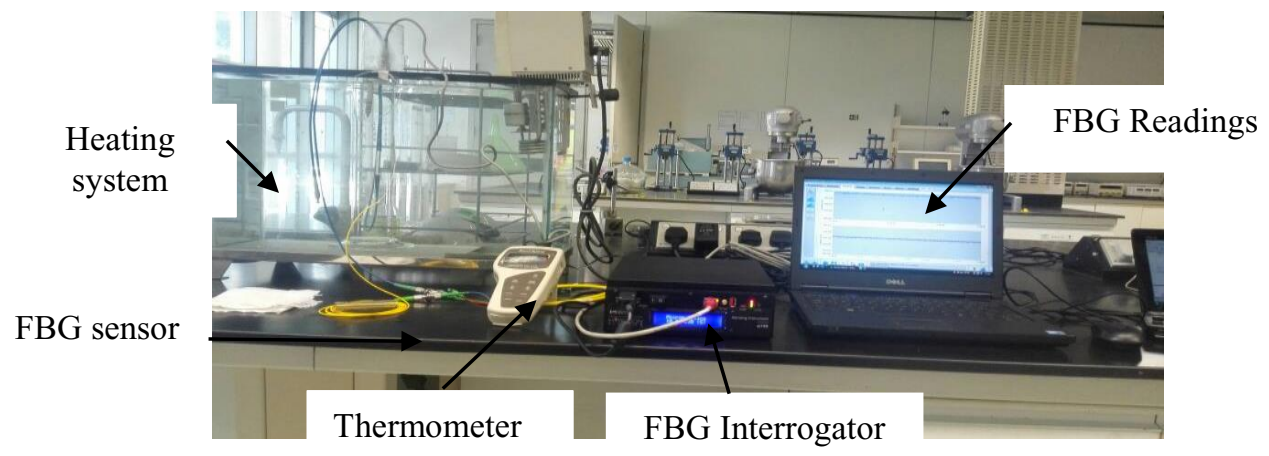

Fig. 2. Experimental setup of temperature calibration test 


\subsection{Strain calibration of FBG sensor}

FBG sensor is calibrated using a setup shown in Fig 3. The apparatus consists of a linear translation stage (Extended linear range ball bearing linear stage manufactured by Newport), which has a right/left hand configuration and stackable to fit any basic translation need. The linear stage is fitted with Vernier micrometre capable of reading up to $13 \mathrm{~mm}$. A customized metal box is fabricated to aid the measurement. Both the linear stage and the metal box are mounted on an optical board at a distance apart.

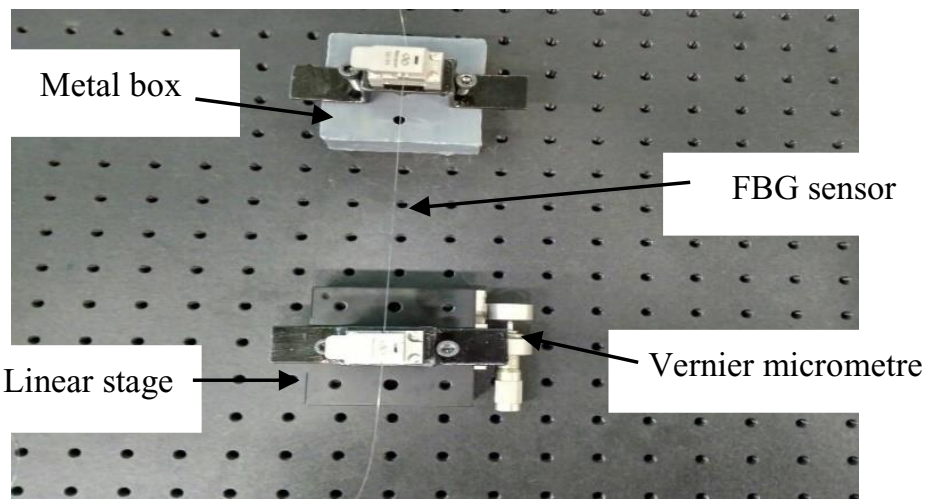

Fig. 3. FBG sensor strain calibration setup

FBG sensor with wavelength of $1570 \mathrm{~nm}$ is fixed between the linear stage and the metal box. When the micrometre fitted on the linear stage is turned, corresponding FBG sensor readings are taken with the aid of interrogator at the frequency of $1 \mathrm{kHz}$. The initial distance between the linear stage and the metal box is $230 \mathrm{~mm}$. FBG sensor can be related to strain using equation 3 , where $k$ is the strain coefficient.

\section{Results and discussion}

The graph of comparison of temperature and averaged wavelength is plotted as shown in Fig 5. It can be observed that both the heating and cooling processes followed the same pattern this implies a very good temperature calibration. The relationship between the wavelength and the temperature is represented by the polynomial equation given in equation 4 .

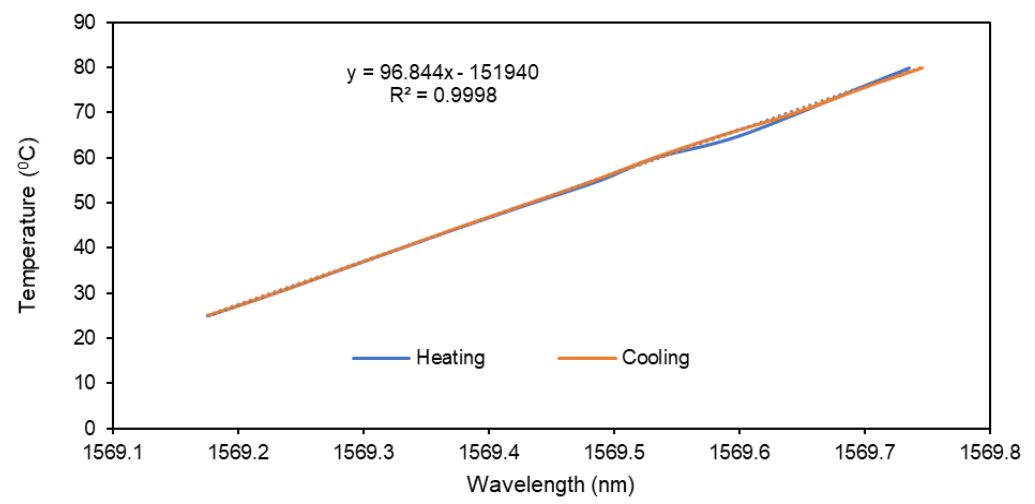

Fig. 5. FBG sensor temperature calibration. 


$$
T=96.844 \lambda_{T}-151940
$$

Where $T$ is the temperature and $\lambda_{T}$ is the FBG wavelength. A graph of temperature measured from the thermometer and that observed using FBG was plotted (Fig. 6) and the $R^{2}$ value was found to be 0.9993 while the slope is unity which is an indication of a very good similarity. Similar result was observed by[13].

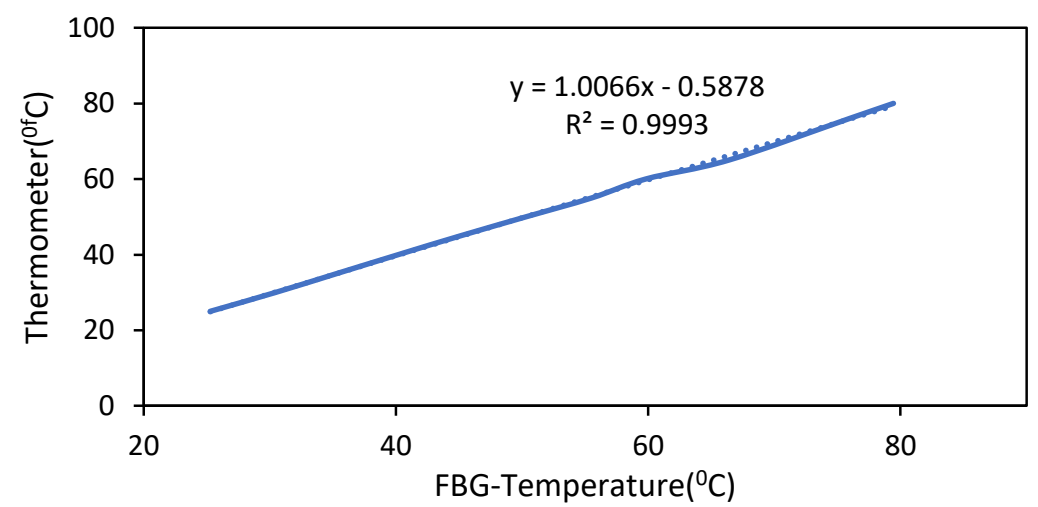

Fig. 6. The relationship between measure temperature and the FBG-temperature

Strain calibration test results is presented in Fig 7. The $R^{2}$ value of 0.999 signifies perfect correlation between the micrometre strain and the average FBG wavelength shift. The slope of change in the wavelength against the strain was found to be approximately 0.77 , which is the value of the strain coefficient. Similar finding was presented by [14], also [11], [15] adopted almost similar coefficient. The variation may be due to the different in fiber or may be fluctuation in temperature during the experiment.

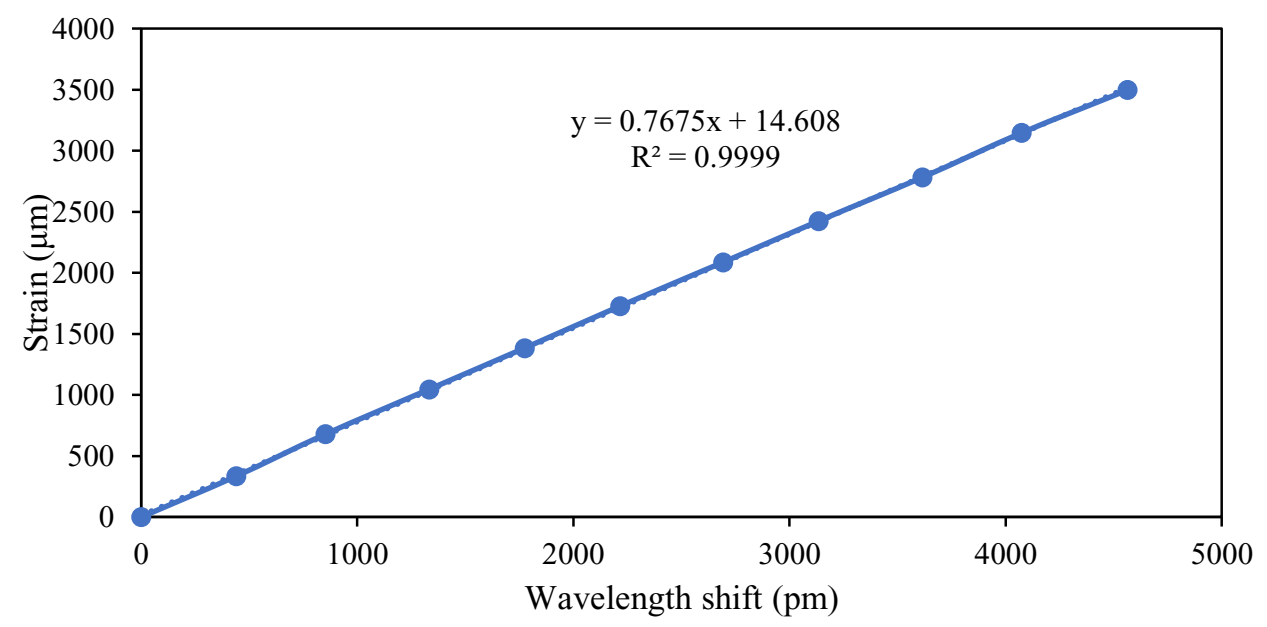

Fig. 7. Strain calibration of FBG sensor.

From the analysis of results, two stain conversion equations can be generated; stain without temperature compensation and strain with temperature compensation. When effect 
of temperature variation is negligible, equation 5 can be adopted. If the temperature variation is significant equation 6 is most suitable.

$$
\begin{gathered}
\varepsilon=0.77\left(\lambda_{B}-\lambda_{B 0}\right) \times 1000=770\left(\lambda_{B}-\lambda_{B 0}\right) \\
\varepsilon=770\left(\lambda_{\varepsilon}-\lambda_{\varepsilon 0}\right)-96.844\left(\left(\lambda_{T}-\lambda_{T 0}\right)\right.
\end{gathered}
$$

The generalized form of the equation can be written as:

$$
\varepsilon=\kappa_{\varepsilon}\left(\lambda_{\varepsilon}-\lambda_{\varepsilon 0}\right)-\kappa_{T}\left(\left(\lambda_{T}-\lambda_{T 0}\right) \times \kappa_{T E}\right.
$$

Where:

$\varepsilon:$ Strain

$\kappa_{\varepsilon}:$ Strain coefficient

$\lambda_{\varepsilon}$ : Current peak wavelength captured by the interrogator

$\lambda_{\varepsilon 0}$ : Initial peak wavelength of the FBG

$\kappa_{T}$ : Temperature coefficient

$\lambda_{T}$ : Current wavelength of temperature designated FBGs captured by the Interrogator

$\lambda_{T 0}$ : Initial wavelength of temperature designated FBGs

$\kappa_{T E}$ : Coefficient of thermal expansion of the material or structure to be monitored

\section{Conclusion}

Simple methods of converting FBG sensor wavelength to temperature and strain are demonstrated. Based on the findings, the temperature coefficient observed can be employed to compensate FBG temperature effect during the subsequent application of the sensor. This simple method can be recommended for determining FBG sensor temperature coefficient.

Also, the strain coefficient obtained showed perfect correlation with micrometre strain. Subsequently, FBG sensor will be employed to measure strain and temperature in the laboratory using the coefficients found as conversion factors.

\section{References}

1. C. Massaroni, P. Saccomandi, and E. Schena, "Medical smart textiles based on fiber optic technology: An overview," J. Funct. Biomater., 6, 2, pp. 204-221 (2015).

2. A. Bin Huang, J. T. Lee, Y. Te Ho, Y. F. Chiu, and S. Y. Cheng, "Stability monitoring of rainfall-induced deep landslides through pore pressure profile measurements," Soils Found., 52, 4, pp. 737-747 (2012).

3. J. Wu, H. Jiang, J. Su, B. Shi, Y. Jiang, and K. Gu, "Application of distributed fiber optic sensing technique in land subsidence monitoring," J. Civ. Struct. Heal. Monit., 5, 5, pp. 587597 (2015).

4. P. Doherty et al., "Field validation of fibre Bragg grating sensors for measuring strain on driven steel piles," Géotechnique Lett., 5, pp. 74-79 (2015).

5. S. Uchida, E. Levenberg, and A. Klar, "On-specimen strain measurement with fiber optic distributed sensing," Measurement: Journal of the International Measurement Confederation, 60. pp. 104-113 (2015).

6. Y. Sun, Q. Li, D. Yang, C. Fan, and A. Sun, "Investigation of the dynamic strain responses of sandstone using multichannel fiber-optic sensor arrays," Eng. Geol., 213 (2016).

7. H. Q. Liao, Z. R. Qiu, and G. H. Feng, "The design of LDF data acquisition system based on LabVIEW," Procedia Environ. Sci., 10, no. Part B, pp. 1188-1192 (2011). 
8. X. Qing, A. Kumar, C. Zhang, I. F. Gonzalez, G. Guo, and F. K. Chang, "A hybrid piezoelectric/fiber optic diagnostic system for structural health monitoring," Smart Mater. Struct., 14, 3 (2005).

9. C. J. Pacheco and A. C. Bruno, A noncontact force sensor based on a fiber Bragg grating and its application for corrosion measurement., Sensors (Basel)., 13, 9, pp. 11476-11489 (2013).

10. D. S. Xu, L. Borana, and J. H. Yin, Measurement of small strain behavior of a local soil by fiber Bragg grating-based local displacement transducers, Acta Geotech., pp. 1-9, (2013).

11. D.-S. Xu, "A New Measurement Approach for Small Deformations of Soil Specimens Using Fiber Bragg Grating Sensors," Sensors, 17, 5, p. 1016 (2017).

12. K. O. Hill and G. Meltz, "Fiber Bragg Grating Technology Fundamentals and Overview," IEEE J. Light. Technol., 15, 8, pp. 1263-1276 (1997).

13. J. and Zhang and Y. Li, Calibration Method for Fiber Bragg Grating Temperature Sensor, Ninth Int. Conf. Electron. Meas. Instruments, pp. 822-825 (2009).

14. P. Boccaccio, R. Donà, and A. Zoccoli, Calibration of a Fiber Bragg Grating as Ultra-Sensitive Strain Gauge, pp. 191-192 (2010).

15. Y. Sun, Q. Li, and C. Fan, "Laboratory core flooding experiments in reservoir sandstone under different sequestration pressures using multichannel fiber Bragg grating sensor arrays," Int. J. Greenh. Gas Control, 60, pp. 186-198 (2017). 